\title{
Carotid access for percutaneous coronary intervention
}

\author{
Matthias De Boulle ${ }^{1}$, Erik Debing ${ }^{1}$, Dries Belsack ${ }^{1}$, and Bert Vandeloo ${ }^{1}$ \\ ${ }^{1}$ Universitair Ziekenhuis Brussel
}

July 24, 2021

\begin{abstract}
Vascular access for percutaneous coronary intervention (PCI) is usually obtained through the radial or femoral, and to lesser extent the brachial or ulnar artery. We describe the transcarotid approach for PCI in a patient with severe peripheral artery disease. No adverse neurological or cardiac events were observed.
\end{abstract}

Title page

Title:

Carotid access for percutaneous coronary intervention

Authors:

Matthias De Boulle, MD a

Erik Debing, $\mathrm{MD}, \mathrm{PhD}^{\text {a }}$

Dries Belsack, MD ${ }^{\mathrm{b}}$

Bert Vandeloo, $\mathrm{MD}^{\mathrm{a}}$

${ }^{\text {a }}$ Centrum voor Hart- en Vaatziekten, Vrije Universiteit Brussel (VUB), Universitair Ziekenhuis Brussel (UZ Brussel), Brussels, Belgium

b Department of Radiology, Vrije Universiteit Brussel (VUB), Universitair Ziekenhuis Brussel (UZ Brussel), Brussels, Belgium

Total word count :

1355

Funding:

None

Disclosure:

All authors have reported that they have no financial disclosure relevant to the content of this paper.

Address for correspondence :

Name: Matthias De Boulle

Address: Universitair Ziekenhuis Brussel, Laarbeeklaan 101, 1090 Jette, Belgium

Email: matthias.deboulle@gmail.com 
Telephone: +32 24776009

Fax: +3224776851

Keywords:

Drug Eluting Stent

Atherosclerosis

Leriche syndrome

Key clinical message

Transcarotid percutaneous coronary intervention is feasible and safe, and can be considered as an ultimate alternative in cases where conventional peripheral vascular access is unavailable.

\section{Abstract}

Vascular access for percutaneous coronary intervention (PCI) is usually obtained through the radial or femoral, and to lesser extent the brachial or ulnar artery. We describe the transcarotid approach for PCI in a patient with severe peripheral artery disease. No adverse neurological or cardiac events were observed.

Text

\section{Introduction}

Vascular access for percutaneous coronary intervention (PCI) is gained via a peripheral artery, mainly the radial or femoral artery. Other less frequently used access sites include the brachial and ulnar artery (1). In this article, we present a patient in which conventional arterial access sites where inaccessible due to severe peripheral artery disease, and where access was obtained at the left carotid artery for PCI of the left anterior descending coronary artery (LAD).

\section{Case report}

A 64-year-old woman, without prior cardiac history, was admitted to the hospital with worsening dyspnea on exertion, orthopnea and swelling of the lower limbs. She had hypercholesterolemia and prediabetes and was an active smoker. Clinical examination showed signs of congestion. A transthoracic echocardiogram revealed a dilated left ventricle with severe left ventricular dysfunction, with a left ventricular ejection fraction of $25 \%$. The diagnosis of congestive heart failure was made. Medical heart failure therapy was initiated.

To rule out underlying coronary artery disease, an invasive coronary angiography was planned. However, vascular access could not be obtained despite several attempts via different arterial access points, both radial and femoral. A computed tomography angiography (CTA) as well as a coronary computed tomography angiography (CCTA) were performed. The CTA showed extensive peripheral artery disease, with a total occlusion of the infrarenal aorta and both common iliac arteries (Leriche Syndrome), a total occlusion of both subclavian arteries, and a critical stenosis of the brachiocephalic artery (Figure 1), explaining the inability to obtain access through conventional access sites. Furthermore, there was a severe stenosis of the left internal carotid artery, and a mild stenosis of the left common carotid artery. However, arterial access could be obtained proximally at the left carotid artery. The CCTA showed extensive coronary calcifications, with a severe stenosis in the LAD, a moderate stenosis in the right coronary artery (RCA) and a moderate stenosis in the left circumflex artery (LCx), and thus justified the need for coronary artery revascularization.

After multidisciplinary Heart Team discussion, involving the vascular surgeon, the decision was made to perform PCI of the LAD via the left carotid artery.

The configuration of the catheterization lab was adjusted to enable carotid access site preparation and PCI (Figure 2). The procedure was performed under general anesthesia. First, the vascular surgeon made the skin incision along the anterior border of the sternocleidomastoid muscle. The subcutaneous tissue and platysma were incised. The sheath over the common carotid artery was opened and the artery encircled with 
a vessel loop. After that, a retrograde puncture of the artery was performed and a guidewire with a 6 French sheath were positioned in the aortic arch under fluoroscopy. To ensure that the sheath stayed fixated, it was sutured to the sternocleidomastoid muscle (Figure 3). A diagnostic coronary angiogram was then made by the interventional cardiologist. The presence of a severe stenosis in the mid segment of the LAD and moderate stenoses in the RCA and LCx were confirmed. PCI was performed, with deployment of a XIENCE Sierra? $2.75 \times 38 \mathrm{~mm}$ drug eluting stent (Abbott, Santa Clara, CA, USA) in the mid LAD. Adequate stent expansion and complete stent apposition were achieved, confirmed by angiography and intravascular ultrasound (IVUS) (Figure 4). Finally, the vascular surgeon removed the sheath, closed the puncture hole with a prolene suture, and sutured the overlying tissues and skin.

The length of the combined procedure was 111 minutes, with a total fluoroscopy time of 14 minutes. The total dose area product (DAP) was $25.311 \mathrm{~Gy} . \mathrm{cm}^{2}$, with a total air kerma (K) of $318 \mathrm{mGy}$. A total of 150 $\mathrm{ml}$ of iodinated contrast was used. During the procedure, 5000 units of unfractionated heparin were given. The patient received dual antiplatelet therapy, consisting of acetylsalicylic acid 80mg and clopidogrel $75 \mathrm{mg}$ once daily.

During the postoperative observation period, the patient showed good vital signs, no chest pain and no neurological symptoms. She was discharged from the hospital the next day.

\section{Discussion}

The transcarotid approach has been described as a viable alternative for vascular access in several other procedures, such as aortic repair interventions (2), cerebral angiography (3), endovascular intervention for acute ischemic stroke $(4,5)$, complex pediatric cardiac interventions $(6,7)$, and transcatheter aortic valve implantation (TAVI) (8-10). One case of a diagnostic coronary catheterization via transcarotid approach has also been described (11). This is, to our knowledge, the first reported adult case of a PCI via transcarotid approach.

In a recent meta-analysis, comparing transcarotid versus transfemoral TAVI, transcarotid TAVI seems to be non-inferior to transfemoral TAVI in terms of overall mortality and short-term neurological complications such as transient ischemic attack and stroke (8). As such, safety for performing transcarotid percutaneous interventions has been demonstrated. Since our current experience is limited to this case alone, we cannot make general conclusions regarding the safety of PCI via transcarotid approach. Nonetheless, our patient experienced no adverse neurological or cardiac events during or after the procedure.

For this procedure, the configuration of the catheterization lab was different than the usual configuration used for PCI via the radial or femoral approach. Apart from this adjusted catheterization lab configuration, the materials used for the PCI were the same as what is normally used, including a standard sheath and standard coronary catheters.

\section{Conclusion}

Our case demonstrates that transcarotid PCI is feasible. The transcarotid approach can be considered as an ultimate alternative in cases where conventional peripheral vascular access for PCI is unavailable.

\section{Acknowledgement}

We thank Margot Allonsius for taking and processing the periprocedural images.

\section{Author contributions}

Matthias De Boulle: literature research, manuscript writing

Eric Debing: manuscript correction and writing

Dries Belsack: data processing, manuscript correction

Bert Vandeloo: literature research, manuscript correction 


\section{References}

1. Kolkailah AA, Alreshq RS, Muhammed AM, Zahran ME, Anas El-Wegoud M, Nabhan AF. Transradial versus transfemoral approach for diagnostic coronary angiography and percutaneous coronary intervention in people with coronary artery disease. Cochrane Database Syst Rev. 2018 Apr 18;4:CD012318.

2. Wee I, Syn N, Choong A. Carotid Access For Aortic Interventions: Genius Or Madness? Vasc Endovasc Rev. 2018 Sep 23;1:38-42.

3. Bauer J, Salazar JL, Sugar O, Pawl RP. Direct percutaneous cerebral angiography in neurosurgical practice. J Neurosurg. 1980 Apr;52(4):525-8.

4. Cilingiroglu M, Hakeem A, Wholey M, Goktekin O. Direct carotid access for endovascular management of acute ischaemic stroke. EuroIntervention J Eur Collab Work Group Interv Cardiol Eur Soc Cardiol. 2017 Oct 13;13(9):e1120-1.

5. Colombo E, Rinaldo L, Lanzino G. Direct carotid puncture in acute ischaemic stroke intervention. Stroke Vasc Neurol. 2020 Jan 29;5(1):71-9.

6. Davenport JJ, Lam L, Whalen-Glass R, Nykanen DG, Burke RP, Hannan R, et al. The successful use of alternative routes of vascular access for performing pediatric interventional cardiac catheterization. Catheter Cardiovasc Interv Off J Soc Card Angiogr Interv. 2008 Sep 1;72(3):392-8.

7. Polat TB. Use of percutaneous carotid artery access for performing pediatric cardiac interventions: Single-center study. Ann Pediatr Cardiol. 2020;13(1):16-24.

8. Wee IJY, Stonier T, Harrison M, Choong AMTL. Transcarotid transcatheter aortic valve implantation: A systematic review. J Cardiol. 2018 Jun 1;71(6):525-33.

9. Folliguet T, Laurent N, Bertram M, Zannis K, Elfarra M, Vanhuyse F, et al. Transcarotid transcatheter aortic valve implantation: multicentre experience in France. Eur J Cardio-Thorac Surg Off J Eur Assoc Cardio-Thorac Surg. 2018 Jan 1;53(1):157-61.

10. Modine T, Lemesle G, Azzaoui R, Sudre A. Aortic valve implantation with the CoreValve ReValving System via left carotid artery access: first case report. J Thorac Cardiovasc Surg. 2010 Oct;140(4):928-9.

11. Lichtenwalter C, Guleserian KJ, Holper EM. Carotid access for cardiac catheterization: revisiting the past for a complex intra-arterial approach. Cardiovasc Revasc Med. 2011 Mar 1;12(2):134.e1-134.e5.

Figures

Figure 1

Computed tomography angiography showing a total occlusion of the infrarenal aorta and both common iliac arteries (arrows), and a total occlusion of both subclavian arteries (arrowheads).

Figure 2

The configuration of the catheterization lab, which allowed for carotid access site preparation and percutaneous coronary intervention.

Figure 3

A perioperative view, showing the left common carotid artery with a 6 French sheath fixated to the sternocleidomastoid muscle.

Figure 4

Angiographic view of the severe mid left anterior descending artery stenosis, before (a) and after (b) percutaneous coronary intervention with implantation of a drug eluting stent. 


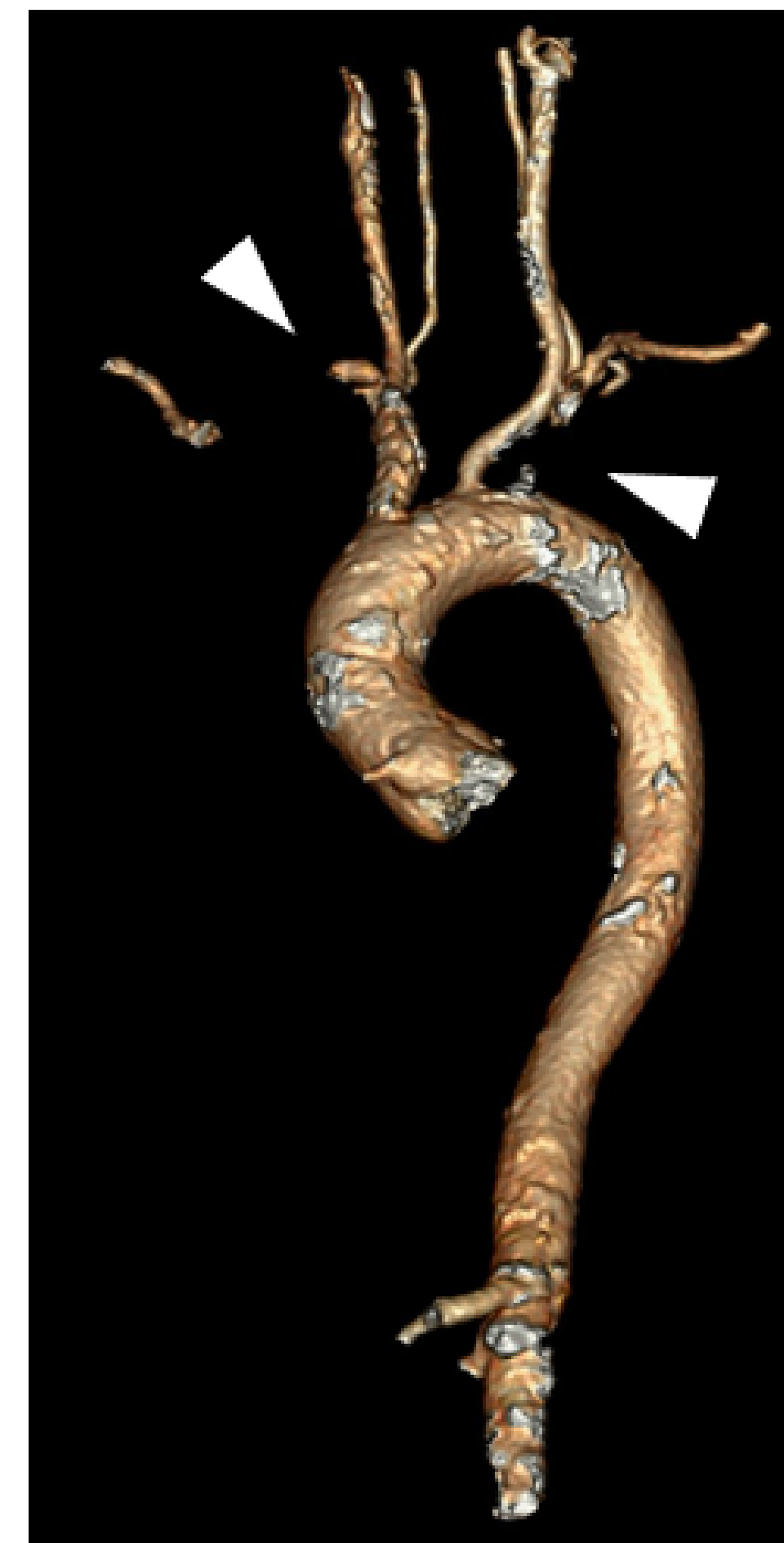



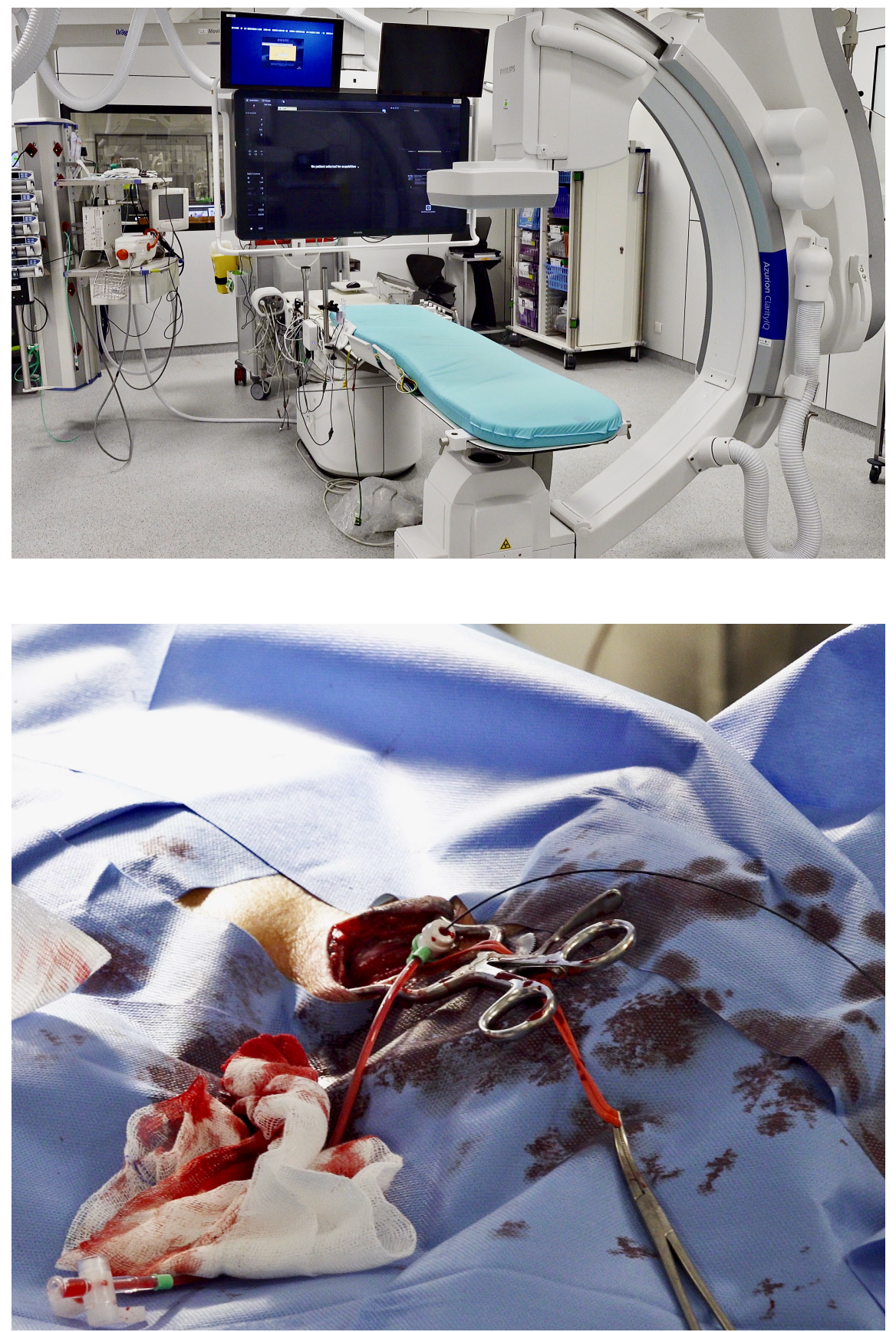


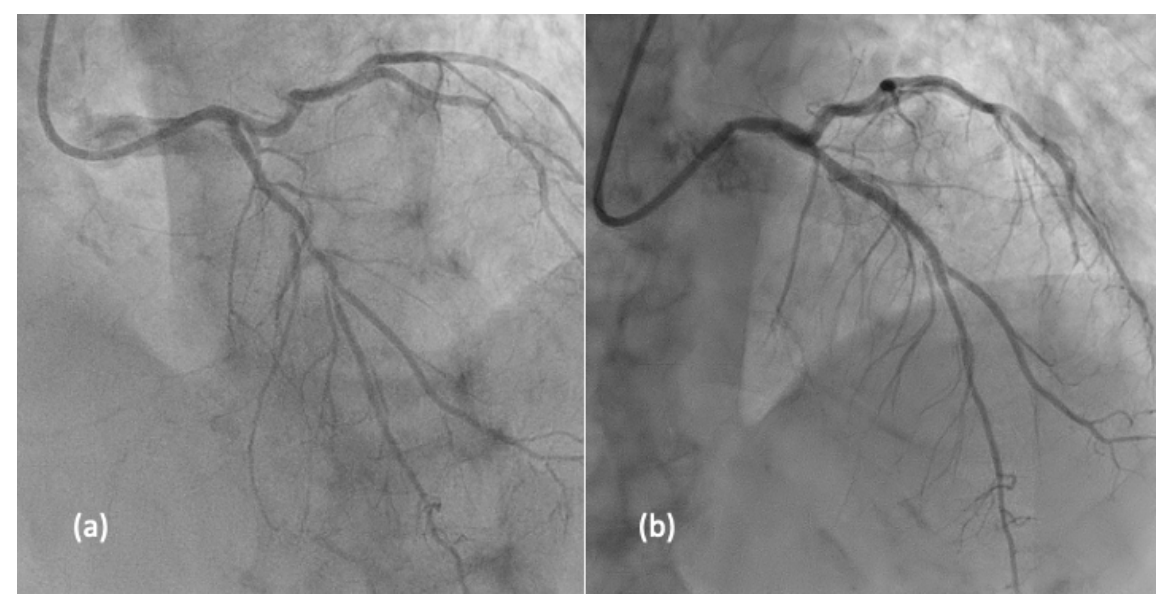

